\title{
Development of a SeaWinds Wind Product for Weather Forecasting
}

\author{
M. Portabella, A. Stoffelen, and J. de Vries \\ Royal Dutch Meteorological Institute (KNMI) \\ Postbus 201, 3730 AE De Bilt, The Netherlands \\ Phone : +31 30 2206827, Fax : +31 302210843
}

\begin{abstract}
SeaWinds data are provided by the Jet Propulsion Laboratory (JPL) at 25-km resolution, whereas most numerical weather prediction (NWP) models use observations at least a 100-km density. We have developed a QuikSCAT wind product at a coarser resolution than the JPL product in order to achieve a more appropriate information content for assimilation in NWP models. This product includes quality control, wind retrieval, and ambiguity removal.
\end{abstract}

\section{INTRODUCTION}

The SeaWinds instrument on QuikSCAT satellite is a conically scanning pencil-beam scatterometer, which in comparison with the fan-beam NASA scatterometer (NSCAT) has the following advantages: higher signal-tonoise ratio, smaller size, and superior coverage.

Severe storms that hit Europe often originate over the North Atlantic Ocean, where sparse meteorological observations are available. As a consequence, the initial stage of severe storms is often poorly analysed and their development poorly predicted. The SeaWinds data coverage is such that developing storms are likely observed, thus depicting their position and amplitude. However, the SeaWinds product as available from Jet Propulsion Laboratory (JPL) is often contaminated by serious inversion problems and ambiguity removal errors and do not provide any meteorological guidance [1]. In such cases, SeaWinds product improvement as described in this report is essential.

SeaWinds data are nominally provided with a sampling of $25 \mathrm{~km}$, whereas most numerical weather prediction (NWP) models use observations at at least a $100-\mathrm{km}$ density. The small-scale structures observed by a scatterometer cannot be well fitted by the relatively broad spatial structure functions, and result in statistical noise in the analyses. For scatterometer data, in order to reduce systematic wind retrieval errors it is better to reduce noise and average backscatter measurements $\left(\sigma^{0}\right)$ to lower resolution before the non-linear wind retrieval process. Here we present a $100-\mathrm{km}$ SeaWinds wind product which is based on three major steps: wind quality control, wind retrieval and ambiguity removal. Applications such as nowcasting, short-range forecasting and NWP assimilation may benefit from this product.

\section{QUALITY CONTROL}

In order to successfully assimilate QuikSCAT data into NWP models, a comprehensive quality control (QC) needs to be done in advance. References [2] and [3] use a method to detect and reject WVCs with poor quality wind information using a Maximum-Likelihood-Estimator-based (MLE) parameter for the European Remote-Sensing Satellite (ERS) scatterometer and NSCAT, respectively. Here, we adapt this method for QuikSCAT.

The MLE indicates how well the backscatter measurements used in the retrieval of a particular wind vector fit the Geophysical Model Function (GMF), which is derived for fair weather wind conditions. A large inconsistency with the GMF results in a large MLE, which indicates geophysical conditions other than those modeled by the GMF, such as rain, confused sea state, or ice, and as such, the MLE provides a good indication for the quality of the retrieved winds.

Collocations with ECMWF winds and SSM/I rain data are used to characterize the MLE-based QC parameter called the normalized residual $(\mathrm{Rn})$. The $\mathrm{QC}$ procedure is defined to achieve the following goals: maximum low-quality data rejection, including rain contamination; and minimum goodquality data rejection. An $\mathrm{Rn}$ threshold is found, which satisfies these goals [4]. The QC is using the MLE information from the JPL $25-\mathrm{km}$ product and therefore applied to the $25-\mathrm{km} \mathrm{WVC}$, prior to the wind inversion at $100-\mathrm{km}$ resolution.

\section{WIND RETRIEVAL}

In order to retrieve winds in a super WVC or SWVC (100$\mathrm{km}$ resolution) coming from $\sigma^{0}{ }_{25}$ measurements of $1625-\mathrm{km}$ WVC's, we consider three different scenarios :

1) Simply use per beam the up to $16 \sigma^{0}{ }_{25}$ values together with the observed noise values of $K_{p o}, K_{p \beta}, K_{p \gamma}$ azimuth and incidence angles from the WVC's, for the valid data in the QuikSCAT BUFR product.

2) Construct a $K_{p}$-averaged backscatter value per beam representative for the SWVC from the up to $16 \sigma^{0}{ }_{25}$. The values of the azimuth and incidence angle are averaged per flavour (beam) for the $16 \mathrm{WVC}$ 's.

3) The same as scenario 2 except that we take the geometric mean of the $16 \sigma_{25}^{0}$ values as the backscatter value representative for the SWVC.

In order to test which of the 3 scenarios gives the optimal wind retrieval, we perform a simulation. We use the JPL 25$\mathrm{km}$ BUFR product rank-1 wind field to simulate the measurements with their appropriate noise. We then perform the inversion at $100-\mathrm{km}$ resolution for the three different scenarios. The JPL rank-1 wind solution closest to the 
centroid of the SWVC is used as truth for validation purposes. We compare the rank-1 solution to the truth. We conclude from the results (not shown) that a SWVC mean $\sigma^{0}{ }_{100}$ based on the geometric mean of the backscatter values of the individual WVC's (scenario 3) gives the best fit between simulated winds and the truth, also in terms of smallest standard deviation of the difference (SD) and biases. Therefore, scenario 3 is chosen to retrieve $100-\mathrm{km}$ winds [1].

\section{AMBIGUITY REMOVAL}

Ambiguity removal (AR) is the process of selecting the wind vector solution at each observation point in a way that results in a spatially and meteorologically consistent wind field. In order to do so, we apply a variational scheme called 2D Variational Ambiguity Removal 2D-VAR [1], which attempts to minimize the cost function

$$
J(\delta \underline{x})=J_{b}+J_{o}^{\text {scat }},
$$

where $J_{b}$ is the background term and $J_{o}^{\text {scat }}$ is the observation term. It uses an incremental formulation with the control variable of wind increments, $\delta \underline{x}=\underline{x}-\underline{x}_{b}$, defined on a rectangular equidistant grid. The control variable $\underline{x}_{b}$ is the background field, which in 2DVAR is an NWP model forecast. The forecast is also used as first guess making the control variable equal to the null-vector at the start of the minimization.

The $J_{b}$ is a quadratic term that contains the inverse of the background error covariance matrix. It penalizes the deviation from the background field. The background error covariances are considered homogeneous and isotropic, i.e. a function of separation distance only. Since the background error covariance matrix is a full (not diagonal) and large matrix, it is expensive to compute its inverse. Therefore, $J_{b}$ is computed in the Fourier domain, where the matrix can be expressed as a diagonal matrix. The $J_{o}^{\text {scat }}$ expresses the misfit between the ambiguous wind vector solutions and the control variable at each observation point. The contribution of the wind solutions (up to four in this case) in each observation point is weighted by a solution probability. This solution probability depends on the angular sector distribution of the retrieved wind directions and the distance to the GMF after inversion. It is assumed that these two pieces of information are independent.

In order to solve the minimization problem, a conjugate gradients method is used, which also requires the gradient of the cost function. After convergence, the control variable vector of wind increments is added to the background field to obtain the wind analysis. The analyzed wind field is a spatially consistent weighted mean of the background field and the observations. It is used to select at each observation point the wind solution ambiguity with the smallest RMSdifference. The selected ambiguity is considered to be the observed wind.

\section{RESULTS}

To assess the quality of the retrieval and the ambiguity removal a comparison was made between the JPL $25-\mathrm{km}$ BUFR wind product thinned to $100-\mathrm{km}$ and the KNMI 100$\mathrm{km}$ wind product. For the comparison, wind speed, wind direction and wind components ( $u$ and $v$ ) were computed for an independent reference (ECMWF 10-m wind) and both the JPL-thinned product and the $100-\mathrm{km}$ KNMI product. Here we only show the results for the comparison of the wind direction component, but similar conclusions can be drawn from the other wind component comparisons.

Figs. 1 and 2 show the two-dimensional histograms of rank1 and selected wind directions, respectively, versus ECMWF wind directions, for JPL-thinned (a) and KNMI 100-km (b) winds.

The distributions in Fig. 1 show some peaks around the lines of 180 degrees deviation, which refer to a directional ambiguity problem. These peaks are significantly less pronounced in fig. $1 \mathrm{~b}$, showing that the ambiguity problem is much reduced in the KNMI product. Moreover, the statistics show a higher SD in fig. 1a compared to fig. 1b. This gives a strong indication that the retrieval at $100-\mathrm{km}$ improves the quality of the rank-1 solution at the ECMWF model resolution. However, if we look at Fig. 2, the direction distribution has been considerably improved by the JPL AR (fig. 2a), while in KNMI (fig. 2b) the 180-degree ambiguity problem slightly remains. The statistics show lower SD in fig. $2 b$ compared to fig. 2a. Therefore, JPL AR works better than KNMI AR.

In fig. 3, we show a typical tropical case with more moderate and low winds. In fig. 3a, the JPL winds show a general preference to blow across-swath and to a lesser degree along-swath. Moreover, some erratic large wind vectors appear that are most likely caused by rain [4]. The KNMI product in fig. $3 \mathrm{~b}$ has cured to a large degree both the directional preference and the erratic speed variations. This is fairly typical in the tropical area, thus indicating an improved performance at low and moderate wind speeds.

Meteorologists at KNMI have been validating the $100-\mathrm{km}$ product for a period of 4 months. They report that in $15.5 \%$ of cases the wind products are considered as essential information, in $48.8 \%$ as important and in $35.7 \%$ of cases rated as of little use. It is apparent that a $100-\mathrm{km}$ scatterometer wind product has added value for synoptic meteorological analysis. We conclude that the KNMI 100-km inverted winds are of better quality than the JPL thinned product. However, work remains to be done in the AR scheme.

\section{ACKNOWLEDGMENT}

The QuiSCAT data are processed at NASA PO.DAAC (JPL) and converted to BUFR format at NOAA. This work was carried out with both EUMETSAT and Dutch BCRS funds. 
a)

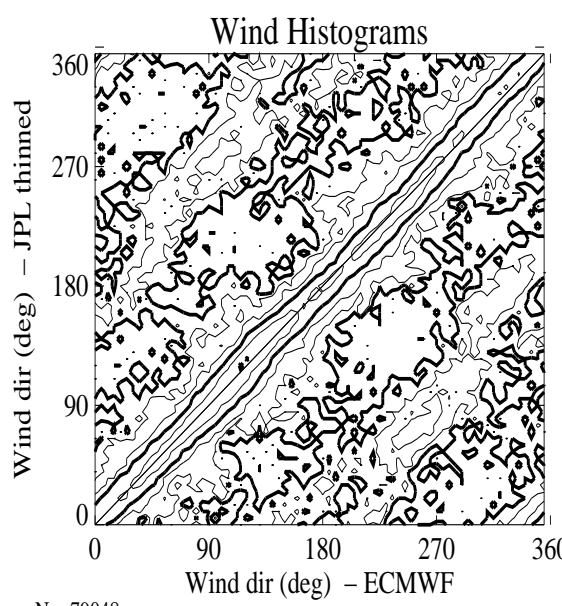

$\mathrm{N}=79048$

$\mathrm{mx}=175.92 \mathrm{my}=175.52$

$\mathrm{m}(\mathrm{y}-\mathrm{x})=-0.40 \mathrm{~s}(\mathrm{y}-\mathrm{x})=48.16$

cor_xy $=0.89$ b)

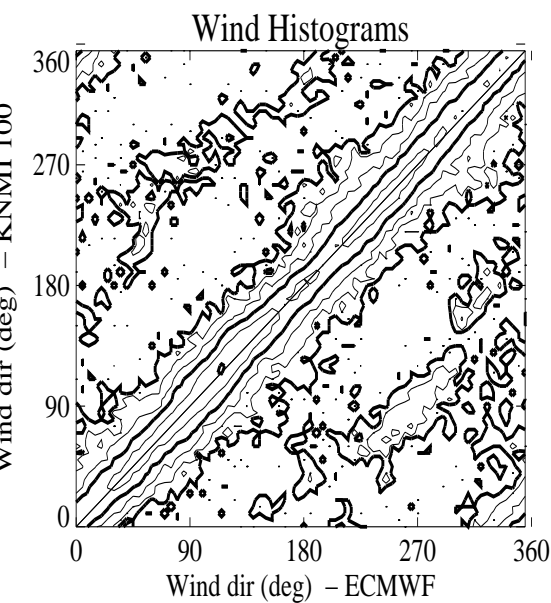

$\mathrm{N}=79482$

$m x=171.06 \mathrm{my}=172.28$

$\mathrm{m}(\mathrm{y}-\mathrm{x})=1.21 \mathrm{~s}(\mathrm{y}-\mathrm{x})=28.26$

cor_xy $=0.96$

Fig. 1. Two-dimensional histograms of JPL-thinned (a) and KNMI 100-km (b) wind directions versus ECMWF $10-\mathrm{m}$ wind directions. $\mathrm{N}$ is the number of data; $\mathrm{mx}$ and $\mathrm{my}$ are the mean directions along $\mathrm{x}$ and $\mathrm{y}$ axis, respectively; $\mathrm{m}(\mathrm{y}-\mathrm{x})$ and $\mathrm{s}(\mathrm{y}-\mathrm{x})$ are the bias and $\mathrm{SD}$, respectively; and cor_xy is the correlation factor.

a)

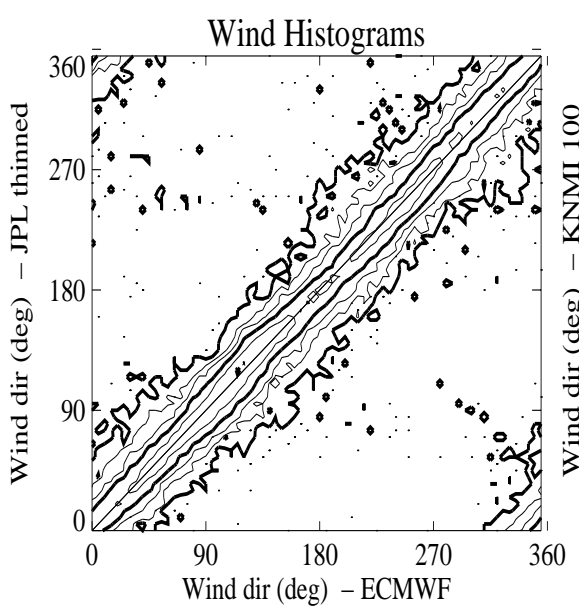

$\mathrm{N}=75523$

$\mathrm{mx}=169.31 \mathrm{my}=169.56$

$\mathrm{m}(\mathrm{y}-\mathrm{x})=0.25 \mathrm{~s}(\mathrm{y}-\mathrm{x})=14.15$

cor_xy $=0.99$ b)

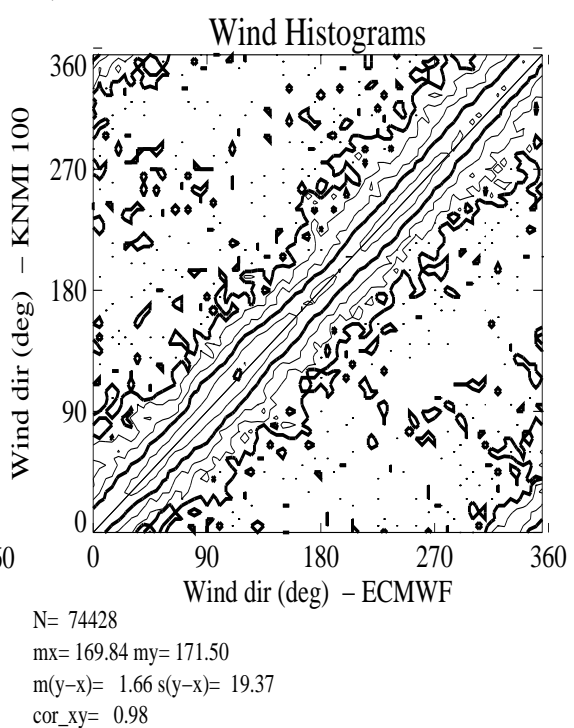

Fig. 2. Same as fig. 1 but for selected instead of rank-1 wind directions.

a)

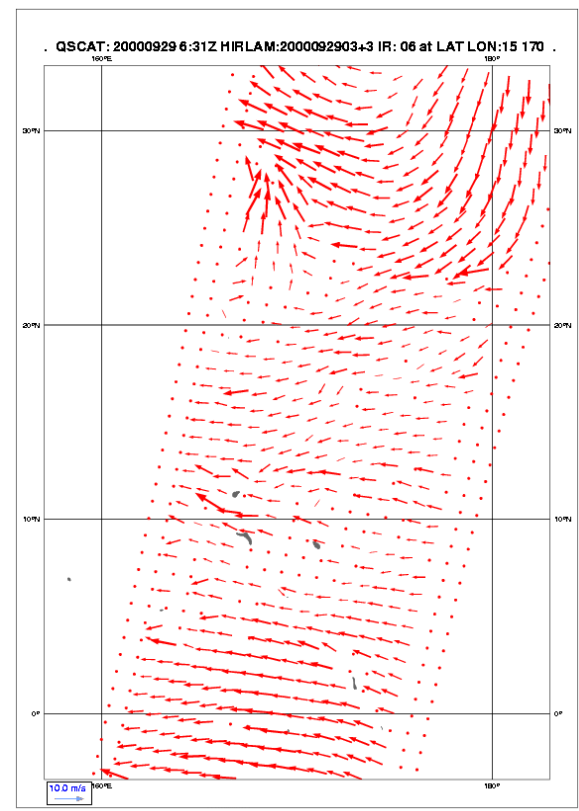

b)

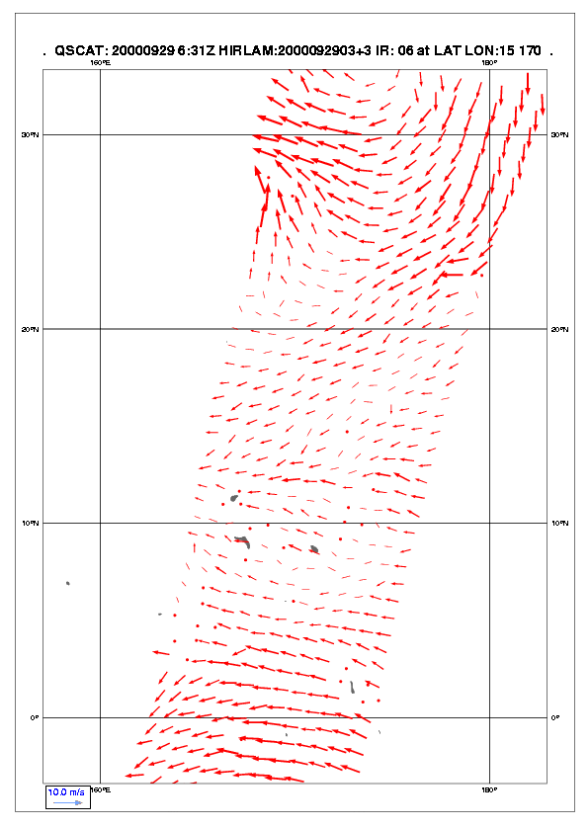

Fig. 3. QuikSCAT JPL-thinned (a) and KNMI $100-\mathrm{km}$ (b) wind fields from September 29 2000 at 06:30 UTC

\section{REFERENCES}

[1] A. Stoffelen, J. de Vries, and A. Voorrips, "Towards the real-time use of Quikscat winds," Beleidscomissie Remote Sensing Report, contract USP-2/00-26, pp. 70, 2001.

[2] A. Stoffelen and D. Anderson, "Scatterometer Data Interpretation: Measurement Space and Inversion," J. Atm. and Ocean. Techn., vol. 14(6), pp. 1298-1313, 1997.
[3] J. Figa and A. Stoffelen, "On the Assimilation of $\mathrm{Ku}-$ band Scatterometer Winds for Weather Analysis and Forecasting," IEEE Trans. on Geoscience and Rem. Sens., Vol. 38(4), pp. 1893-1902, 2000.

[4] Portabella, M., and Stoffelen, A., "Rain Detection and Quality Control of SeaWinds," J. Atm. and Ocean. Techn., in press. 